\title{
Electronic brachytherapy as adjuvant therapy for early stage breast cancer: a retrospective analysis
}

\author{
William C Dooley' \\ James CWurzer ${ }^{2}$ \\ Mohamed Megahy ${ }^{3}$ \\ Gary Schreiber ${ }^{4}$ \\ Tapan Roy ${ }^{5}$ \\ Gary Proulx 6 \\ Susan Laduzinsky ${ }^{7}$ \\ Steven Lane ${ }^{8}$ \\ James Dalzell ${ }^{9}$ \\ Kambiz Dowlatshahi ${ }^{10}$ \\ Dwelvin Simmons"I \\ John P Thropay ${ }^{12}$ \\ Harish Ahuja ${ }^{13}$ \\ Peter Beitsch ${ }^{14}$ \\ Randall W Holt ${ }^{15}$ \\ Charles A Lee ${ }^{16}$ \\ 'Oklahoma University Health Sciences \\ Center, Oklahoma City, OK, USA; \\ ${ }^{2}$ Atlanticare, Egg Harbor Township, NJ, \\ USA; ${ }^{3}$ Maryville Oncology, Maryville, IL, \\ USA; ${ }^{4}$ Swedish Covenant Hospital, Chicago, \\ IL, USA; ${ }^{5}$ Cape Girardeau Cancer Center, \\ Cape Girardeau, MO, USA; ${ }^{6}$ Exeter Hospital, \\ Exeter, NH, USA; ${ }^{7}$ St. Elizabeth's Medical \\ Center, Swansea, IL, USA; ${ }^{8}$ Brockton \\ Hospital, Brockton, MA, USA; ${ }^{9} \mathrm{Nazha}$ \\ Cancer Center, Northfield, NJ, USA; \\ ${ }^{10}$ Rush University Medical Center, Chicago, \\ IL, USA; "'Florida Radiation Oncology \\ Group, Jacksonville, FL, USA; ${ }^{12}$ Beverly \\ Oncology and Imaging Center, Montebello, \\ CA, USA; ${ }^{13}$ Aspirus Cancer Center, Wausau, \\ WI, USA; ${ }^{14}$ Dallas Surgical Group, Dallas, \\ TX, USA; ${ }^{15}$ Pacific Crest Medical Physics, \\ Chico, CA, USA; ${ }^{16}$ Gulf Coast Cancer \\ Treatment Center, Panama City, FL, USA
}

Correspondence: William C Dooley

The G Rainey Williams Chair of Surgical

Oncology, University of Oklahoma

Health Sciences Center, Oklahoma City,

OK 73104, USA

$\mathrm{Tel}+$ I 405 27I 7867

Fax + I 405 27| 4443

Email william-dooley@ouhsc.edu
This article was published in the following Dove Press journal:

OncoTargets and Therapy

II January 2011

Number of times this article has been viewed

Purpose: This multicenter, retrospective study evaluated treatment and clinical outcomes of patients with early stage breast cancer who received adjuvant high-dose rate (HDR) electronic brachytherapy (EBT) treatment post-lumpectomy using the Axxent ${ }^{\circledR}$ EBT system. Dosimetric data from the EBT treatment plans were compared with those based on iridium-192 HDR brachytherapy.

Material and methods: Medical records of 63 patients with early stage breast cancer (Tis, T1a, $\mathrm{T} 1 \mathrm{~b}, \mathrm{~T} 1 \mathrm{c}$, and T2) who were treated post-lumpectomy with EBT alone or in combination with external beam radiation therapy were reviewed. The prescribed EBT dose was 34 Gy (10 fractions over 5 days, 3.4 Gy each) to $1 \mathrm{~cm}$ from the balloon surface. Dosimetry data from 12 patients were compared with these of treatment plans using an iridium-192 source prepared for the same 12 patients.

Results: The majority of patients $(90.5 \%)$ were older than 50 years and had one or more risk factors for breast cancer (80.6\%). Tumor sizes were $0.1 \mathrm{~cm}$ to $3.5 \mathrm{~cm}$ (mean $1.3 \mathrm{~cm}$ ). Median follow-up was 7 months ( 1 to 18 months) post-EBT. Balloon applicators were implanted 0 to 85 days (mean 13.4 days) post-lumpectomy/re-excision. The most common adverse events were erythema, rash dermatitis, and pain or breast tenderness. No recurrences were reported. Dosimetric analyses demonstrated comparable target coverage, increased high-dose regions, and a significantly reduced dose to the ipsilateral breast and lungs as well as the heart with EBT as compared with the iridium-192 treatment plans.

Conclusion: This retrospective, multicenter study showed that postsurgical adjuvant radiation therapy for early stage breast cancer can be administered using the EBT system with similar toxicity outcomes to those reported with iridium-192 brachytherapy. EBT offers a convenient, portable, nonisotope alternative to HDR brachytherapy using iridium-192.

Keywords: electronic brachytherapy, breast cancer, radiation therapy

\section{Introduction}

An estimated 192,370 new cases of invasive and 54,000 noninvasive breast cancer were diagnosed in women in the United States in 2009. ${ }^{1}$ Breast-conserving surgery (BCS) followed by radiation therapy is a standard treatment option for early stage breast cancer. ${ }^{2}$ However, some patients elect to undergo mastectomy due to the length of radiation treatment after BCS, which can be 6 to 7 weeks of daily treatments. This time commitment can represent insurmountable logistical difficulties with regard to work or family responsibilities, leaving the patient to choose a more time-efficient, but debilitating, surgical outcome. ${ }^{3-5}$ Accelerated partial breast irradiation (APBI) offers patients a substantially reduced treatment time by directing radiation to the portion of the breast tissue most likely to experience recurrence, the tissue surrounding the 
tumor cavity. APBI may reduce exposure to radiation of nontarget tissue such as the heart and lungs when compared with whole breast irradiation ${ }^{6-8}$ and has demonstrated equivalent control rates to whole breast irradiation following BCS in several studies. ${ }^{9-12}$

The majority of APBI techniques utilize an iridium192 source with an average energy of $383 \mathrm{kV}$. This form of radiation requires a high-dose-rate (HDR) afterloader unit and a shielded room, which is a financial burden to smaller institutions or clinics and can present scheduling challenges at higher volume centers. Electronic brachytherapy (EBT) utilizes a $50 \mathrm{kV}$ X-ray source that does not require a wellshielded radiation vault or an HDR afterloader unit, and the device can be easily moved from one procedure room to the next once regulatory requirements are met, allowing flexibility in scheduling patients. EBT also does not require the storage and handling of isotopes. ${ }^{13}$ Consequently, EBT may allow additional treatment centers to provide APBI to patients, increasing patients' access to APBI and overall treatment choices.

The Axxent ${ }^{\circledR}$ EBT system (Xoft, Inc., Sunnyvale, CA) has been utilized for APBI in the United States since 2007. The $\mathrm{X}$-ray source is inserted through a balloon applicator, which is implanted post-BCS into the tumor cavity. A controller powers the source and allows dose sculpting by moving the source through a range of dwell positions with dwell times chosen to achieve the prescription dose to the target tissue. In the initial, prospective, multicenter study of EBT in women with Stage 1-2 breast cancer, a prescription dose of 34 Gy was successfully delivered in 10 fractions over 5 days using this EBT system in 42 of 44 patients treated. ${ }^{7}$ A reduced dose (33.96 and 30.60 Gy) was delivered in the other two patients. To better understand how HDR EBT is utilized outside the context of a study protocol, a retrospective multicenter study was designed to collect data on treatment delivery and clinical outcomes in patients who received adjuvant radiation therapy with the EBT system for early stage breast cancer following BCS.

\section{Materials and methods Eligibility}

The medical records of 63 patients with early stage breast cancer who were treated post-lumpectomy using the EBT system between April 2007 and November 2009 were reviewed. Records of patients treated with EBT alone or in combination with external beam radiation therapy (EBRT) were included. Records of patients previously or currently enrolled in other prospectively enrolling EBT studies were excluded.
An Institutional Review Board for each participating center approved the protocol prior to commencing data collection. The data were collected on case report forms and confidentiality was maintained at all times.

\section{Data collection}

Data on patient demographics and tumor characteristics, treatment, and follow-up were collected. For study sites willing to participate in a dosimetric comparison substudy, the EBT treatment plans from this retrospective study were compared to treatment plans created for the same patients for use with an iridium-192 source. An independent dosimetrist completed this subanalysis. The primary objective of this retrospective chart review is to report a real-world experience on the treatment and acute outcomes of patients treated with EBT, and provide a comparative dosimetric analysis of EBT with HDR therapy using iridium-192.

\section{Definitions}

Adverse events were recorded. Tumors were graded based on the American Joint Committee on Cancer (AJCC) classification where Tis = carcinoma in situ, $\mathrm{T} 1 \mathrm{a}=$ tumor $>0.1 \mathrm{~cm}$ but $\leq 0.5 \mathrm{~cm}$ in its greatest dimension, $\mathrm{T} 1 \mathrm{~b}=$ tumor $>0.5 \mathrm{~cm}$ but $\leq 1 \mathrm{~cm}$ in its greatest dimension, $\mathrm{T} 1 \mathrm{c}=$ tumor $>1 \mathrm{~cm}$ but $\leq 2 \mathrm{~cm}$ in its greatest dimension, T2 $=$ tumor $>2 \mathrm{~cm}$ but $\leq 5 \mathrm{~cm}$ in its greatest dimension, and Tmic $=$ microinvasion $=0.1 \mathrm{~cm}$ in its greatest dimension. The histopathologic grades were based on $\mathrm{G} 1$ = well differentiated, G2 = moderately differentiated, G3 = poorly differentiated, G4 = undifferentiated, Gx = grade cannot be assessed, and NA = grade not available.

\section{Materials}

The EBT system (Axxent ${ }^{\circledR}$, Xoft, Inc., Sunnyvale, CA) consists of the X-ray source, the balloon applicator, and the controller. The X-ray source comprises an X-ray tube in a multilumen catheter that allows cooling fluid to circulate over the tube. A sterile, disposable, single-use balloon applicator functions as a guide for the X-ray source. The EBT controller provides power to the X-ray source and allows the X-ray source to be linearly translated within the applicator. The translation, or pullback movement, of the X-ray source within the applicator is designed to provide a conformal dose to the 1-cm prescription point.

\section{Treatment planning}

The prescription dose and brachytherapy treatment plans were prepared individually for each patient based on computed 
tomography (CT) scans. BrachyVision ${ }^{\mathrm{TM}}$ treatment planning software (Varian Medical Systems, Palo Alto, CA) or Plato treatment planning software (Nucletron, Columbia, MD) were used at all centers. The measurement of the radiation dose (dosimetry) was based on Gy, which is the unit of absorbed radiation SI. Delivered dose was computed using TG-43 parameters specific to this source. ${ }^{14} \mathrm{~A}$ balloon applicator was implanted following BCS and remained in place for the 5-day treatment period.

\section{Dosimetry}

A retrospective analysis was conducted with the three-dimensional CT image data from 12 patients using the BrachyVision treatment planning system to compare the dosimetry of the EBT source with that of an iridium-192 source (GammaMedPlus; Varian Medical Systems). The actual EBT treatment plans with the dwell times and positions used to treat each patient were included in the subanalysis. CT scans of the treated breast from the 12 patients were digitally contoured to identify the balloon applicator, the clinical target volume (CTV), ipsilateral breast tissue, heart, lungs, skin, and ribs. A planning target volume (PTV) was obtained from each CTV from the surface of the CTV out to $1 \mathrm{~cm}$ from the balloon into the surrounding tissue. A comparison set of plans was created by replacing the EBT source with an iridium-192 source. The dwell times for the iridium-192 plans were optimized to deliver 34 Gy in 10 fractions to at least $95 \%$ of the PTV (D95), while minimizing the maximal dose to the PTV. Dose-volume histograms (DVH) for both source types were used for the dose comparison. The DVHs of each contoured structure on both plans were compared using the percent of the PTV receiving $90 \%, 100 \%, 150 \%, 200 \%$, and $300 \%$ of the prescribed dose (V90, V100, V150, V200, and V300, respectively). Similarly, comparisons were made for each plan type for the percent of the ipsilateral breast volume receiving $50 \%$ of the prescribed dose (Breast V50), the percent of the lung volume receiving $30 \%$ of the prescribed dose (Lung V30), and the percent of the heart volume receiving $5 \%$ of the prescribed dose (Heart V5). The maximum calculated doses to the skin and rib were compared. Lastly, the percent of the heart volume receiving more than 18 Gy for EBT and 20 Gy for iridium-192 were compared.

\section{Statistics}

Data were entered into an Access database (Microsoft, Redmond, WA) via paper case report forms. Analyses of the data were performed using SAS statistical analysis software (version 9.1.3; SAS Institute, Cary, NC). The $n$ (number of observations) and proportion was reported for both the treatment success and acute outcome endpoints. For summary statistics of continuous variables, the $n$, mean, standard deviation, and range were presented. Variables that reflect categories of information were described using proportions and frequencies.

\section{Results}

Records at 13 clinical sites from 63 patients with Stage 1-2 breast cancer were reviewed (Table 1). Nearly all were aged 50 years and over $(90.5 \%)$ and had one or more risk factors for breast cancer $(80.6 \%)$, including family history of breast or other cancers, history of breast lesions, post-menopausal, age at menarche, parity status, weight, and age. Tumor sizes ranged from $0.1 \mathrm{~cm}$ to $3.5 \mathrm{~cm}$ with a mean of $1.3 \mathrm{~cm} \pm 0.8 \mathrm{~cm}$. All patients had undergone BCS. Fifteen patients at seven study sites had re-excision due to positive margins (Table 1),

Table I Patient characteristics

\begin{tabular}{|c|c|}
\hline & $\mathbf{N}(\%)$ \\
\hline Number of patients & $63(100 \%)$ \\
\hline \multicolumn{2}{|l|}{ Age range } \\
\hline $30-39$ & I (I.6\%) \\
\hline $40-49$ & $5(7.9 \%)$ \\
\hline $50-59$ & 7 (II.1\%) \\
\hline $60-69$ & $30(47.6 \%)$ \\
\hline $70-79$ & $10(15.9 \%)$ \\
\hline $80-89$ & $9(14.3 \%)$ \\
\hline $90-99$ & I (I.6\%) \\
\hline \multicolumn{2}{|l|}{ AJCC classification } \\
\hline Tis & $16(25.4 \%)$ \\
\hline Tla & $9(14.3 \%)$ \\
\hline Tlb & $8(12.7 \%)$ \\
\hline Tlc & $19(30.2 \%)$ \\
\hline $\mathrm{T} 2$ & 7 (II.1\%) \\
\hline Not documented & $4(6.3 \%)$ \\
\hline \multicolumn{2}{|c|}{ Histopathologic grade } \\
\hline GI & $23(36.5 \%)$ \\
\hline $\mathrm{G} 2$ & $18(28.6 \%)$ \\
\hline G3 & $15(23.8 \%)$ \\
\hline Gx & $2(3.2 \%)$ \\
\hline Not documented & $5(7.9 \%)$ \\
\hline \multicolumn{2}{|l|}{ Tumor size $(\mathrm{cm})$} \\
\hline Mean \pm SD & $1.3 \pm 0.8$ \\
\hline Median & 1.2 \\
\hline Range & $0.1-3.5$ \\
\hline \multicolumn{2}{|c|}{ Re-excision due to positive margins } \\
\hline Yes & $15(22.2 \%)$ \\
\hline No & $48(77.8 \%)$ \\
\hline
\end{tabular}


and two patients had positive margins at the time of treatment (Table 2). The median follow-up was 7 months, (range 1 month to 18 months) after the last EBT treatment.

Balloon applicators were implanted a mean of 13.4 days (range 0 to 85 days) after the lumpectomy or last re-excision. Balloon sizes and their corresponding fill volumes are listed in Table 2. In one patient, an inflated balloon did not conform adequately to the tumor cavity; however, a second attempt at implantation was successful in this patient. In another patient, the balloon deflated a few hours after the implantation and was subsequently replaced. The applicator was returned to the device manufacturer, and it was determined that the deflation was caused by exposure of the applicator to a sharp object at the time of insertion.

The prescribed dose of radiation was 34 Gy at a depth of $1 \mathrm{~cm}$ from the balloon surface, which was to be delivered twice daily in 10 fractions of 3.4 Gy each. This prescription was delivered in 62 of 63 patients. For one patient, the CT

Table 2 Treatment summary

\begin{tabular}{ll}
\hline \multicolumn{2}{l}{ Time (days) from lumpectomy or re-excision to applicator } \\
placement \\
Mean \pm SD & $13.4 \pm 14.1$ \\
Median & 9.0 \\
Range & $0-85$ \\
Time (days) from applicator placement to first fraction \\
Mean \pm SD & $3.8 \pm 1.7$ \\
Median & 4.0 \\
Range & $0-7$ \\
Balloon surface to skin distance (mm) & \\
Mean \pm SD & $14.2 \pm 6.6$ \\
Median & 11.8 \\
Range & $7.0-36.0$ \\
Margin pathology status & \\
Negative & $61(96.8 \%)$ \\
Positive & $2(3.2 \%)$ \\
Balloon applicator size & \\
$3-4 \mathrm{~cm}$ & $16(25.4 \%)$ \\
$4-5 \mathrm{~cm}$ & $39(61.9 \%)$ \\
$5-6 \mathrm{~cm}$ & $4(6.3 \%)$ \\
Not Documented & $4(6.3 \%)$ \\
&
\end{tabular}

Balloon applicator sizes and fill volume

\begin{tabular}{lllll}
\hline & $\mathbf{3 - 4} \mathbf{~ c m}$ & $\mathbf{4 - 5} \mathbf{~ c m}$ & $\mathbf{5 - 6} \mathbf{~ c m}$ & $\begin{array}{l}\text { Not } \\
\text { Documented }\end{array}$ \\
\hline $\mathrm{N}$ & 16 & 38 & 4 & 4 \\
\multicolumn{2}{l}{ Saline volume (cc) } & & & \\
Mean \pm SD & $38.2 \pm 5.5$ & $51.3 \pm 11.2$ & $91.3 \pm 33.3$ & $51.7 \pm 10.7$ \\
Median & 36.5 & 50.0 & 100.0 & 50.6 \\
Min & 30.0 & 35.0 & 45.0 & 40.0 \\
Max & 45.0 & 95.0 & 120.0 & 65.5 \\
\hline
\end{tabular}

Notes: 'One patient in the 4-5-cm group did not have saline volume documented; ${ }^{2}$ Four patients had saline volume documented without a balloon applicator size. Abbreviations: SD, standard deviation; min, minimum; max, maximum. scan showed that the balloon applicator was directly adjacent to the rib causing the rib to be within the PTV. Due to this proximity to the rib, the physician determined that a boost treatment followed by EBRT was the optimal course of treatment. The prescribed dose was 7.5 Gy, 2.5 Gy for three fractions, and EBRT was subsequently administered at 45 Gy in 25 fractions. The patient would have undergone 33 EBRT treatments without prior boost treatments.

Adverse events were recorded and if they were rated, the ratings were reported based on the Common Terminology Criteria for adverse events version 3 (CTCv3). Thirtyfive out of 63 patients experienced adverse events. The adverse events recorded during the treatments included one occurrence for each of the following: grade 1 rash, grade 1 nausea, grade 1 fatigue, and grade 1 rash dermatitis related to radiation. There was one occurrence of grade 2 rash dermatitis related to radiation. The most common adverse events reported during the follow-up period were erythema, rash dermatitis associated with radiation, and pain or breast tenderness. Twenty-six of the adverse events in 16 patients were not graded in severity. All adverse events are listed in Table 3. One occurrence of a grade 4 rash dermatitis associated with chemoradiation was reported as a radiation recall reaction in a patient for whom doxorubicin hydrochloride and cyclophosphamide administration commenced 10 days after the final fraction. The rash was almost fully healed 18 days later, which was 28 days after the final fraction. The 6-month visit showed hypopigmentation and the last visit at 10-month follow-up showed erythema that was treated with hydrocortisone cream. One occurrence of a grade 3 moist desquamation occurred in one patient and had improved to erythema grade 1 at 4 months after the final fraction. No other severe reactions were reported as related to treatment. One patient died from Stage 4 pancreatic cancer 294 days after their last EBT treatment.

The occurrence of a seroma was reported in five patients (7.9\%). Out of the five patients, two seromas required drainage and the other three were observed. Four patients (6.3\%) experienced infections and were treated with oral antibiotics. One patient was thought to have an infection and underwent debridement of tissue. Upon examination of the debrided tissue, it was determined that this patient $(1.6 \%)$ had fat necrosis and not an infection. No other cases of fat necrosis were reported.

Individual treatment plans were prepared for each patient prior to beginning EBT. For the dosimetric comparison substudy, separate treatment plans for an iridium-192 source were 
Table 3 Number (\%) of adverse events that were possibly, probably, or definitely related to treatment during treatment or follow-up for all 63 patients

\begin{tabular}{|c|c|c|c|c|c|c|}
\hline \multirow[t]{2}{*}{ Adverse event } & \multirow{2}{*}{$\begin{array}{l}\mathbf{N}(\%) \text { of } \\
\text { adverse events }\end{array}$} & \multicolumn{4}{|c|}{ CTC grade' (n) } & \multirow{2}{*}{$\begin{array}{l}\text { Not } \\
\text { graded (n) }\end{array}$} \\
\hline & & I & 2 & 3 & 4 & \\
\hline Erythema & $14(22.2 \%)$ & 7 & I & 0 & 0 & 6 \\
\hline Rash dermatitis associated with radiation ${ }^{2}$ & $10(15.9 \%)$ & 4 & 4 & 0 & I & 2 \\
\hline Pain/breast tenderness & $10(15.9 \%)$ & 6 & I & 0 & 0 & 3 \\
\hline Rash/dry desquamation & $5(7.9 \%)$ & 2 & 0 & 0 & 0 & 3 \\
\hline Hypopigmentation & $5(7.9 \%)$ & 5 & 0 & 0 & 0 & 0 \\
\hline Hyperpigmentation & $5(7.9 \%)$ & 2 & 0 & 0 & 0 & 3 \\
\hline Seroma & $5(7.9 \%)$ & I & I & 0 & 0 & 3 \\
\hline Infection & $4(6.3 \%)$ & 0 & 2 & 0 & 0 & 2 \\
\hline Moist desquamation ${ }^{3}$ & $3(4.8 \%)$ & 0 & 2 & I & 0 & 0 \\
\hline Pruritis & $2(3.2 \%)$ & 1 & 0 & 0 & 0 & I \\
\hline Fatigue & $2(3.2 \%)$ & I & 0 & 0 & 0 & I \\
\hline Ulceration of treated breast & $2(3.2 \%)$ & 0 & 2 & 0 & 0 & 0 \\
\hline Skin sensitivity & I (I.6\%) & 0 & 0 & 0 & 0 & I \\
\hline Fat necrosis & I (I.6\%) & 0 & 0 & 0 & 0 & I \\
\hline Telangiectasia & I (I.6\%) & 0 & I & 0 & 0 & 0 \\
\hline Fibrosis & I (I.6\%) & I & 0 & 0 & 0 & 0 \\
\hline Induration & I (I.6\%) & I & 0 & 0 & 0 & 0 \\
\hline
\end{tabular}

Notes: 'Common Terminology Criteria (CTC) version 3 grade provided if reported; ${ }^{2}$ One event reported as a grade 4 radiation recall was resolved 2.5 months after radiation treatment; ${ }^{3}$ One event reported as a grade 3 moist desquamation was resolved 4 months after radiation treatment.

prepared based on the EBT treatment plans from 12 patient records in this study, with identical PTVs (mean and SD $104.9 \pm 9.6 \mathrm{~mL}$, range $86.0-123.0 \mathrm{~mL}$ ). The prescribed doses were identical (34 Gy) except for the one patient only treated for three fractions (2.5 Gy per fraction, 7.5 Gy total) as described above. The active treatment lengths for EBT vs iridium-192 were identical for 10 of the 12 patients, ranging from $2.5 \mathrm{~cm}$ to $4.5 \mathrm{~cm}$, with two cases requiring an additional dwell point for iridium-192 to achieve optimal PTV dose coverage. The DVH metrics for PTV and organ dose coverage for the two sets of treatment plans are compared in Table 4. The percent of the PTV receiving $100 \%$ of the prescribed dose (V100) was not statistically different between the two treatment modalities. The V150, V200, and V300 were significantly higher $(P<0.05)$ for EBT than iridium-192. The V90 was lower for EBT than iridium-192. The maximum calculated dose to the skin and rib were not significantly different between the two sources. The Breast V50, Lung V30, and Heart V5 were significantly lower with EBT as compared with iridium-192. Of the 12 patients with reviewed dosimetry, no patient received more than $12 \mathrm{~Gy}$ (total dose) to any part of the heart with EBT, while one patient would have received as much as 21.0 Gy to the heart with an iridium-192 plan with $1 \%$ of the volume of the left ventricle receiving at least $20.3 \mathrm{~Gy}$. The patient with fat necrosis had a V150 of $44.6 \mathrm{~mL}$ and a V200 of $19.5 \mathrm{~mL}$.

\section{Discussion}

This retrospective, multicenter study represents an analysis of acute toxicity in patients treated with EBT as postsurgical adjuvant therapy for early stage breast cancer and provides a comparative dosimetric analysis of the EBT treatment plans with those based on an iridium-192 HDR source in a subgroup of patients. The study population of this retrospective study included a broader range of patients and greater variation in the treatment regimens than would be seen in a prospective study, which would specify patient selection criteria and a treatment protocol. A previous study of this EBT system treated 44 patients with tumor sizes up to $2.8 \mathrm{~cm},{ }^{7}$ whereas this study enrolled patients with slightly larger tumor sizes of up to $3.5 \mathrm{~cm}$ and included two patients with positive margins at the time of treatment. The prescribed dose of radiation, 34 Gy to a depth of $1 \mathrm{~cm}$ beyond the balloon surface, was delivered in 62 of 63 patients. As part of a clinical study program in which 176 patients have been treated with EBT, 7,15 this study demonstrates that APBI can be delivered utilizing a technology that provides benefits to the patients and the healthcare providers. The EBT technology can be stored without shielding, and treatment can be conducted in a standard patient room with minimal shielding. This provides a benefit to patients, who have greater accessibility to radiation treatment and may be more compliant with completing adjuvant radiation treatment. Patient scheduling may be facilitated 
Table 4 Dosimetry comparison: outcomes with electronic brachytherapy (EBT) and iridium-192

\begin{tabular}{|c|c|c|c|}
\hline Mean \pm SD & $\begin{array}{l}\text { Iridium-192 } \\
(n=12)\end{array}$ & $\begin{array}{l}\text { EBT } \\
(n=12)\end{array}$ & $\begin{array}{l}P \text {-value } \\
-\end{array}$ \\
\hline Mean PTV dose in Gy & $22.3 \pm 14.4$ & $27.7 \pm 16.6$ & - \\
\hline Range & II.9-45.4 & $|3|-5 \mid .4$. & - \\
\hline V90 in $\mathrm{mL}$ & $96.4 \pm 3.3$ & $93.7 \pm 4.7$ & $P<0.05$ \\
\hline Range & $90.0-99.8$ & 84.9-98.7 & - \\
\hline VI00 in $\mathrm{mL}$ & $87.2 \pm 6.1$ & $87.2 \pm 6.0$ & NS \\
\hline Range & $78.8-94.9$ & $78.3-94.5$ & - \\
\hline VI50 in $\mathrm{mL}$ & $30.0 \pm 6.1$ & $46.3 \pm 6.3$ & $P<0.05$ \\
\hline Range & $20.3-38.1$ & $37.0-56.8$ & - \\
\hline $\mathrm{V} 200 \mathrm{in} \mathrm{mL}$ & $6.5 \pm 3.2$ & $22.3 \pm 5.1$ & $P<0.05$ \\
\hline Range & $0-11.2$ & $14.5-30.3$ & - \\
\hline $\mathrm{V} 300$ in $\mathrm{mL}$ & 0 & $3.8 \pm 2.4$ & $P<0.05$ \\
\hline Range & 0 & $0-7.8$ & - \\
\hline Maximum skin & $33.3 \pm 7.9$ & $33.7 \pm 10.5$ & NS \\
\hline \multicolumn{4}{|l|}{ dose in Gy } \\
\hline Range & $20.0-47.0$ & $16.0-48.0$ & - \\
\hline Maximum rib dose & $26.9 \pm 17.8$ & $29.7 \pm 27.3$ & NS \\
\hline \multicolumn{4}{|l|}{ in Gy } \\
\hline Range & $4.0-59.0$ & I.0-83.0 & - \\
\hline Ipsilateral breast & $217.0 \pm 48.7$ & $141.8 \pm 44.0$ & $P<0.05$ \\
\hline \multicolumn{4}{|l|}{ V50 in $\mathrm{mL}$} \\
\hline Range & 147.0-299.0 & $96.0-225.0$ & - \\
\hline Ipsilateral lung & $23.3 \pm 30.6$ & $8.0 \pm 12.9$ & $P<0.05$ \\
\hline \multicolumn{4}{|l|}{$\mathrm{V} 30$ in $\mathrm{mL}$} \\
\hline Range & $0-90.5$ & $0-36.0$ & - \\
\hline Heart V5 in $\mathrm{mL}$ & $41.5 \pm 29.6$ & $13.1 \pm 20.9$ & $P<0.05$ \\
\hline Range & I.0-82.I & $0-57.3$ & - \\
\hline
\end{tabular}

Abbreviations: PTV, planning target volume; SD, standard deviation.

since the device can be easily moved from one procedure room to the next.

Treatment was well tolerated and the adverse events recorded in this study are generally within the range of grade and frequency reported in other EBT studies, ${ }^{7,15}$ as well as in studies of iridium-192 brachytherapy with a similar duration of follow-up. ${ }^{16}$ The infection rate was $6.3 \%$, which is within the range of $2.9 \%$ to $11.4 \%$ previously reported with $\mathrm{EBT}^{7,15}$ and within the range of $4 \%$ to $16 \%$ previously reported with iridium-based balloon brachytherapy (Ir-BT). ${ }^{16-20}$ Fat necrosis was reported in one patient at 406 days post treatment. Previous studies have shown a correlation between fat necrosis and dose hot spots with interstitial brachytherapy. ${ }^{21,22}$ Wazer et al reported a mean V150 of $69 \mathrm{~mL}$ and V200 of $22 \mathrm{~mL}$ in patients with fat necrosis. ${ }^{21}$ In this study, the mean V150 and V200 were $46 \mathrm{~mL}$ and $22 \mathrm{~mL}$, respectively, for the dosimetric substudy population and $45 \mathrm{~mL}$ and $20 \mathrm{~mL}$, respectively, for the patient with fat necrosis. Beitsch et al reported one-year follow-up data on 37 patients treated with EBT, none of whom had fat necrosis. ${ }^{15}$ Mehta et al reported two cases of fat necrosis in a study population of 44 patients with follow-up times of up to 18 months. ${ }^{7}$ The mean V150 and V200 were $52 \mathrm{~mL}$ and $24 \mathrm{~mL}$, respectively, for the study population. It remains to be seen whether additional reports of fat necrosis will occur as these patients reach 2 years of follow-up. In the future, it will be interesting to compare the rates of fat necrosis from these EBT studies with a currently enrolling study that has limited the V150 to $50 \mathrm{~mL}$ and the V200 to $10 \mathrm{~mL}$ for $\mathrm{IrBT}^{23}$ Five-year follow-up data from the initial IrBT study showed fat necrosis in 4 of $43(9.3 \%)$ patients at 11, 14, 42, and 63 months. Three-year follow-up data from an IrBT registry reported fat necrosis in 22 of 1,440 (1.5\%) patients. ${ }^{24}$

Skin toxicity of grade 4 occurred in one patient who had initiated treatment with chemotherapeutic agents, cyclophosphamide and doxorubicin hydrochloride, within 10 days of the completion of the final EBT fraction. The skin toxicity was almost fully healed at 18 days after the final fraction and resolved at 2.5 months following the final fraction. Previous studies have suggested that careful selection and timing of administration of chemotherapeutic agents must be considered for patients receiving radiation therapy. As with iridium-192 APBI treatments, radiation recall may occur in patients who receive cytotoxic chemotherapeutic agents following APBI, particularly in the presence of thin skin bridges or other factors that could result in an increased dose to the skin. ${ }^{7,25}$ Appropriate patient selection and the timing of administration of cytotoxic chemotherapeutic agents in relation to the radiation therapy treatments, as with patients being treated with iridium-192, must be taken into account when treating patients with EBT.

EBT may also be used in combination with EBRT. In this study, one patient received EBT treatment as a boost dose ( $7.5 \mathrm{~Gy}$ ) followed by EBRT (45 Gy), which provided an optimal treatment for this patient whose tumor cavity was adjacent to a rib. The use of EBT for a boost dose decreased the required number of EBRT treatments for this patient from 33 to 25 fractions. EBT has been used as a boost dose and as sole radiation therapy intra-operatively with surgical staff remaining in the operating room during irradiation; one-year data from the single-center intraoperative radiation therapy (IORT) study demonstrated good cosmesis with no recurrences. ${ }^{26} \mathrm{~A}$ multicenter IORT study randomized patients to whole breast EBRT or intra-operative EBT as sole therapy or as a boost dose prior to EBRT and demonstrated good control rates at 4 years of followup. ${ }^{27}$

Comparative treatment plans based on an iridium192 source were prepared using data from the EBT treatment plans for 12 patients in this study. The required number of dwell positions varied between the two treatments in two 
of 12 cases due to the different inherent dose distributions produced by each source, and this variation was not considered to be clinically relevant. As the plans were designed specifically to deliver a desired dose to the PTV, no difference in V100 would be expected; however, differences were noted with other variables that are consistent with other comparisons of these two types of brachytherapy. ${ }^{6,8,13}$ Hot spots within the PTV were more common with EBT as shown by the V150, V200, and V300 values. However, doses to normal tissue in the ipsilateral breast, lung, and heart were significantly less with EBT as compared to iridium-192. Skin doses in the subanalysis were not significantly different when comparing the EBT group to the Iridium group. With an $11.8 \mathrm{~mm}$ median skin bridge margin (for all 63 patients), which is beyond the normalized PTV prescription point of $1 \mathrm{~cm}$, significant differences would not be expected. The results of this dosimetric comparison are similar to those reported in an IrBT study with a dosimetric substudy comparing the actual IrBT data with EBT treatment plans created for the same patients. ${ }^{6}$ The similarities in outcomes provide confirmation of the value of generating hypothetical treatment plans from data used to create actual treatment plans. The clinical relevance of the differences in dosimetry between an electronic source and an iridium source remain to be seen; however, the potential for clinical relevance may provide an additional factor to consider in the decision about the methodology of radiation therapy for each patient.

The dose to the heart is of concern as a correlation has been shown in the literature between diminished myocardial perfusion over 6 to 24 months and the percent of the left ventricle (LV) that receives a threshold dose over 25 Gy with standard 2 Gy per day EBRT. ${ }^{28}$ Marks et al reported that at 6 and 12 months postradiation, patients with $<1 \%$ of the LV receiving this threshold dose had a $4 \%$ and $12 \%$ incidence of perfusion defect. ${ }^{28}$ For patients with $1 \%$ to $5 \%$ of the LV above the threshold dose, the defect rate at 6 and 12 months was $22 \%$ and $27 \%$, respectively. The threshold dose will be different for hypo-fractionated APBI where the equivalent LV dose threshold for an iridium-192 5-day APBI schedule is $20.3 \mathrm{~Gy}^{29}$ Using the same method as Garza et al, ${ }^{29}$ and assuming a relative biological effectiveness value of 1.2 for $50 \mathrm{kV}$ X-rays, the LV threshold dose for APBI with EBT is 18.7 Gy. For EBT, no patient was at risk for cardiac perfusion effects; by contrast, one of 12 patients with iridium-192 would have been borderline at-risk with $1 \%$ of the LV over the equivalent 20.3 Gy threshold. Determining a specific risk for a $1 \%$ volume of $L V$ radiation out of the $1 \%$ to $5 \%$ volume cohort is not possible. As succinctly noted by Mille, a clinical treatment methodology which minimizes dose to any critical organ is desirable, particularly for the heart. ${ }^{8}$

\section{Conclusion}

This retrospective, multicenter study showed that postsurgical adjuvant radiation therapy for early stage breast cancer can be administered using the EBT system with similar toxicity outcomes to those reported with iridium-192 brachytherapy. Dosimetric analyses demonstrated comparable target coverage, increased high dose regions, and a significantly reduced dose to the ipsilateral breast and lungs as well as the heart with EBT as compared with the iridium-192 treatment plans. EBT offers a convenient, portable, nonisotope alternative to HDR brachytherapy using iridium-192.

\section{Acknowledgments}

The authors wish to thank all research staff who supported this research. The authors would like to acknowledge Leslie Todd, medical writer, and Kathy L Warner, biostatistician, for their assistance with this manuscript.

\section{Disclosure}

Randall Holt is a medical physics director and paid consultant for Xoft, Inc. Charles Lee was compensated for his time required to create the treatment plans. Peter Beitsch is a co-principal investigator of a prospectively enrolling early stage breast cancer study funded by Xoft, Inc. This retrospective study was funded by Xoft, Inc.

\section{References}

1. National Cancer Institute Website. 2010. Available from: http://www. cancer.gov/cancertopics/types/breast. Accessed Jun 162010.

2. National Comprehensive Cancer Network Website. 2010. Available from: http://www.nccn.org/index.asp. Accessed Mar 32010.

3. Pawlik TM, Buchholz TA, Kuerer HM. The biologic rational for and emerging role of accelerated partial breast irradiation for breast cancer. J Am Coll Surg. 2004;199(3):479-492.

4. Schroen AT, Brenin DR, Kelly MD, Knaus WA, Slingluff CL Jr. Impact of patient distance to radiation therapy on mastectomy use in early-stage breast cancer patients. J Clin Oncol. 2005;23(28): 7074-7080.

5. Athas WF, Adams-Cameron M, Hunt WC, Amir-Fazli A, Key CR. Travel distance to radiation therapy and receipt of radiotherapy following breast conserving surgery. J Natl Cancer Inst. 2000;92(3): 269-271.

6. Dickler A, Kirk MC, Seif N, et al. A dosimetric comparison of MammoSite high-dose-rate brachytherapy and Xoft Axxent electronic brachytherapy. Brachytherapy. 2007;6(2):164-168.

7. Mehta VK, Algan O, Griem KL, et al. Experience with an electronic brachytherapy technique for intracavitary accelerated partial breast irradiation. Am J Clin Oncol. 2010;33(4):327-335.

8. Mille MM, Xu G, Rivard MJ. Comparison of organ doses for patients undergoing balloon brachytherapy of the breast with HDR 192-Ir or electronic sources using monte carlo simulations in a heterogeneous human phantom. Med Phys. 2010;37(2):662-671. 
9. Vicini FA, Baglan KL, Kestin LL, et al. Accelerated treatment of breast cancer. J Clin Oncol. 2001;19(7):1993-2001.

10. King TA, Bolton JS, Kuske RR, Fuhrman GM, Scroggins TG, Jiang $\mathrm{XZ}$. Long term results of wide-field brachytherapy as the sole method of radiation therapy after segmental mastectomy for $\mathrm{T}(\mathrm{is}, 1,2)$ breast cancer. Am J Surg. 2000;180(4):299-304.

11. Wazer DE, Berle L, Graham R, et al. Preliminary results of a phase I/II study of HDR brachytherapy alone for T1/T2 breast cancer. Int $J$ Radiat Oncol Biol Phys. 2002;53(4):889-897.

12. Vicini, FA, Kestin L, Chen P, Benitez P, Goldstein NS, Martinez A. Limited-field radiation therapy in the management of early-stage breast cancer. J Natl Cancer Inst. 2003;95(16):1205-1211.

13. Dickler A, Kirk MC, Coon A, et al. A dosimetric comparison of Xoft Axxent electronic brachytherapy and iridium-192 high-dose-rate brachytherapy in the treatment of endometrial cancer. Brachytherapy. 2008;7(4):351-354.

14. Rivard MJ, Davis SD, DeWerd LA, Rusch TW, Axelrod S. Calculated and measured brachytherapy dosimetry parameters in water for the Xoft Axxent X-Ray Source: an electronic brachytherapy. Med Phys. 2006;33(11):4020-4032.

15. Beitsch, PD, Patel RR, Lorenzetti JD, et al. Post-surgical treatment of early-stage breast cancer with electronic brachytherapy: an intersociety, multicenter brachytherapy trial. Onco Targets Ther. 2010; 3: 211-218.

16. Keisch M, Vicini F, Kuske RR, et al. Initial clinical experience with the MammoSite breast brachytherapy applicator in women with early-stage breast cancer treated with breast-conserving therapy. Int J Radiat Oncol Biol Phys. 2003;55(2):289-293.

17. Haley M, Beriwal S, Heron DE, et al. MammoSite accelerated partial breast irradiation: a single-institution outcomes analysis with two years of follow-up. Brachytherapy. 2009;8(1):9-13.

18. Chao KK, Vicini FA, Wallace M, et al. Analysis of treatment efficacy, cosmesis, and toxicity using the MammoSite breast brachytherapy catheter to deliver accelerated partial-breast irradiation: the William Beaumont hospital experience. Int J Radiat Oncol Biol Phys. 2007; 69(1):32-40.

19. Richards GM, Berson AM, Resigno J, et al. Acute toxicity of high dose rate brachytherapy with the MammoSite applicator in patients with early-stage breast cancer. Ann Surg Oncol. 2004;11(8):739-746.
20. Zannis V, Beitsch PD, Vicini F, et al. Descriptions and outcomes of insertion techniques of a breast brachytherapy balloon catheter in 1,403 patients enrolled in the American Society of Breast Surgeons MammoSite breast brachytherapy registry trial. Am J Surg. 2005; 190(4):530-538.

21. Wazer DE, Kaufman S, Cuttino L, DiPetrillo T, Arthur DW. Accelerated partial breast irradiation: an analysis of variables associated with late toxicity and long-term cosmetic outcome after high-dose-rate interstitial brachytherapy. Int J Radiat Oncol Biol Phys. 2006;64(2):489-495.

22. Dickler A, Seif N, Kirk MC, et al. A dosimetric comparison of MammoSite and ClearPath high-dose-rate breast brachytherapy devices. Brachytherapy. 2009;8(1):14-18.

23. Radiation Therapy Oncology Group. National Surgical Adjuvant Breast and Bowel Project (NSABP) protocol B-39 and Radiation Therapy Oncology Group (RTOG) protocol 0413. 2005 Mar 21. http://www. rtog.org/members/protocols/0413/0413.pdf. Accessed Oct 222010.

24. Vicini FA, Beitsch PD, Quiet CA, et al. Three-year analysis of efficacy, cosmesis, and toxicity by the American Society of Breast Surgeons MammoSite breast brachytherapy registry trial in patients with accelerated partial breast irradiation (APBI). Cancer. 2008;112(4): 758-766.

25. Vicini FA, Beitsch PD, Quiet CA, et al. First analysis of patient demographics, technical reproducibility, cosmesis, and early toxicity: results of the American Society of Breast Surgeons MammoSite breast brachytherapy registry trial. Cancer. 2005;104(6):1138-1148.

26. Ivanov O, Dickler A, Lum BYF, Pellicane JV, Francescatti DS. Twelve-month follow-up results of a trial utilizing Axxent electronic brachytherapy to deliver intraoperative radiation therapy for early-stage breast cancer. Ann Surg Oncol. 2010 Aug 25. [Epub ahead of print].

27. Vaidya JS, Joseph DJ, Tobias JS, et al. Targeted intraoperative radiotherapy versus whole breast radiotherapy for breast cancer (TARGIT-A trial): an international, prospective, randomized, non-inferiority phase 3 trial. Lancet. 2010;376(9735):91-102.

28. Marks LB, Yu X, Prosnitz RG, et al. The incidence and functional consequences of RT-associated cardiac perfusion defects. Int J Radiat Oncol Biol Phys. 2005;63(1):214-223.

29. Garza R, Albuquerque K, Sethi A. Lung and cardiac tissue doses in left breast cancer patients treated with single-source breast brachytherapy compared to external beam tangent fields. Brachytherapy. 2006;5(4): 235-238.
OncoTargets and Therapy

\section{Publish your work in this journal}

OncoTargets and Therapy is an international, peer-reviewed, open access journal focusing on the pathological basis of all cancers, potential targets for therapy and treatment protocols employed to improve the management of cancer patients. The journal also focuses on the impact of management programs and new therapeutic agents and protocols on

\section{Dovepress}

patient perspectives such as quality of life, adherence and satisfaction The manuscript management system is completely online and includes a very quick and fair peer-review system, which is all easy to use. Visit http://www.dovepress.com/testimonials.php to read real quotes from published authors. 